\title{
Ultrasound Detection of Epicardial Adipose Tissue Combined With Ischemic Modified Albumin in the Diagnosis of Coronary Heart Disease
}

\author{
Jiandong Xiao, ${ }^{1,2}$ Yuli Lu, ${ }^{1}$ Xinchun Yang ${ }^{2}$ \\ ${ }^{1}$ Department of Cardiology, Beijing Chaoyang Hospital, Capital Medical University, Beijing, China; ${ }^{2}$ Department of Cardiology, \\ Hengshui People's Hospital, Hengshui, Hebei, China
}

\section{ABSTRACT}

Objective: To evaluate the value of epicardial adipose tissue (EAT) thickness combined with serum ischemic modified albumin (IMA) concentration in the diagnosis of coronary heart disease.

Methods: A total of 180 patients with coronary heart disease from May 2017 to December 2018 were selected as the case group. After the examination of Judkins coronary angiography, they were grouped according to the results of angiography, including 60 patients in a single-vessel group, 60 patients in a double-vessel group, and 60 patients in a multi-vessel group. Sixty healthy people with physical examinations at our hospital at the same time were selected as the control group. All selected participants were tested for epicardial adipose tissue thickness by echocardiography and serum IMA concentration by albumin cobalt ion binding test.

Results: The EAT thickness and IMA concentration in the single-vessel disease group, double-vessel disease group, and multi-vessel disease group significantly were higher than those in the control group $(P<.01)$. The ROC curve shows that the sensitivity is $53.33 \%$ and the specificity is $87.6 \%$, when the EAT thickness is $6.12 \mathrm{~mm}$. The sensitivity is $76.0 \%$ and the specificity is $72.3 \%$, when the serum IMA concentration is $72 \mathrm{U} / \mathrm{L}$. When the two were combined, the sensitivity was $40.15 \%$ and the specificity was $96.89 \%$. In the parallel test, the sensitivity was $88.3 \%$ and the specificity was $65.44 \%$.

Conclusion: Ultrasound detection of EAT thickness and serum IMA concentration has certain reference value for the diagnosis of coronary heart disease. Combined diagnosis can significantly improve the diagnosis rate and accuracy of coronary heart disease.

\section{INTRODUCTION}

As we all know, the early diagnosis of coronary heart disease has a great impact on the treatment effect. At the same time, the diagnosis of asymptomatic coronary heart disease

Received April 15, 2020; accepted fune 4, 2020.

Correspondence: Yang Xinchun, Department of Cardiology, Beijing Chaoyang Hospital, Capital Medical University, Beijing, China; 8 Gongren Tiyuchang Nanlu, Chaoyang District, Beijing, 100020; +86-15031828120 (e-mail: yangxinchun_2018@sina.com). is more difficult, which may lead to a missed diagnosis and delay the optimal treatment timing [Wang 2010]. Although coronary angiography and coronary CTA can determine the degree of coronary stenosis, there are deficiencies such as expensive, traumatic, and radiation risks, which are difficult for census recipients to accept. How to find the low-cost, non-invasive, non-radiative, early diagnosis of coronary heart disease signs and targets has become a hot spot in current coronary heart disease research [Yan 2016]. In recent years, studies have found that epicardial adipose tissue (EAT) closely is related to the progression of coronary atherosclerotic plaque, which is an independent risk factor for coronary heart disease [Wang 2014]. Ischemia modified albumin (IMA) can be detected in the early stage of acute myocardial ischemia or myocardial necrosis, becoming a biochemical marker for early diagnosis of myocardial ischemia [Gurumurthy 2014]. All the subjects underwent coronary angiography to understand the coronary artery condition, and the combined detection of EAT thickness and IMA concentration was performed to analyze its value in the early clinical diagnosis of coronary heart disease.

\section{MATERIALS AND METHODS}

Ethics approval: This study was approved by the ethics committee. All patients provided written informed consent.

Study patients: One-hundred eighty patients with coronary heart disease from May 2017 to December 2018 were selected as the study group, including 98 males and 82 females, age 40-81. The average age was $55.53 \pm 8.86$ years old. All patients were examined by Judkins coronary angiography. At least one coronary artery stenosis was $\geq 50 \%$ in the right coronary artery, left main trunk, anterior descending or circumflex branch and its main branches, in line with the diagnostic criteria for coronary heart disease developed by the World Health Organization. In the singlevessel disease group, coronary stenosis was $\geq 50 \%$. In the double-vessel disease group, the stenosis of any two coronary lesions was $\geq 50 \%$. In the multi-vessel disease group, the stenosis of three coronary lesions was $\geq 50 \%$. At the same time, according to the principle of gender and age matching, 60 healthy people were examined by Judkins coronary angiography. These individuals had normal coronary arteries and were selected as the control group; this group included 33 males and 27 females, age 40-79. The average 
Table 1. Comparison of clinical baseline data of study patients

\begin{tabular}{|c|c|c|c|c|c|c|}
\hline Male/Female & $33 / 27$ & $98 / 82$ & $33 / 27$ & $32 / 28$ & $33 / 27$ & .689 \\
\hline Hypertension & $24(40.0 \%)$ & $65(36.11 \%)$ & 22 & 21 & 22 & .301 \\
\hline Total cholesterol $(\mathrm{mmol} / \mathrm{L})$ & $4.65 \pm 0.83$ & $5.34 \pm 0.96^{*}$ & $5.54 \pm 0.68$ & $5.32 \pm 0.98$ & $5.25 \pm 1.33 * *$ & .026 \\
\hline Triglyceride $(\mathrm{mmol} / \mathrm{L})$ & $1.45 \pm 0.56$ & $1.79 \pm 0.47 *$ & $1.73 \pm 0.51$ & $1.78 \pm 0.58$ & $1.89 \pm 0.64 *$ & .043 \\
\hline Low-density lipoprotein (mmol/L) & $4.22 \pm 0.42$ & $4.23 \pm 0.69$ & $4.14 \pm 0.64$ & $4.16 \pm 0.81$ & $4.20 \pm 0.88$ & .352 \\
\hline High density lipoprotein (mmol/L) & $1.15 \pm 0.56$ & $1.10 \pm 0.22$ & $1.18 \pm 0.27$ & $1.13 \pm 0.33$ & $1.06 \pm 0.57$ & .622 \\
\hline
\end{tabular}

Note: compared with the control group, $* P<.05, * * P<.01$

age was $56.66 \pm 7.56$ years old. The case groups and control group were comparable in data, such as gender and age $(P>.05)$. Enrolled patients did not have pericardial diseases or any pericardial-related surgical history. Also excluded were diseases, such as cardiac insufficiency, organic valvular heart disease, severe arrhythmia, tumor, extreme exhaustion and cachexia, and severe liver and kidney dysfunction.

EAT thickness measurement: All patients in the case groups and control group were measured for EAT thickness, using the US GE Vivid 7 color Doppler ultrasound scanner, and the tests were performed by the same physician. The examinee took the left lateral position, applied a heart probe with a frequency of 1.7 to $3.4 \mathrm{MHz}$, scanned the left vertebral long axis section of the sternum, and used the aortic annulus as a positioning marker to display the EAT tissue in front of the right ventricular free wall. At the end of the stop frame, frozen image, vertical aortic annulus, the thickness of the EAT tissue was measured at the thickest part of the right atrioventricular sulcus in front of the right ventricular free wall. A total of five cardiac cycles were measured and averaged [Xia 2011].

Determination of serum IMA concentration: All subjects immediately were admitted to the hospital with $5 \mathrm{ml}$ of venous blood and centrifuged at $3000 \mathrm{rpm}$ for 15 minutes. The serum IMA concentration was determined by the albumin-cobalt ion binding assay (ACB). The kit was provided by Changsha Fukang Technology Co., Ltd.

Statistical processing: Statistical analysis was performed using SPSS 20.0 software. The EAT thickness and IMA concentration of the measurement data were expressed as t-test. The comparison between the two groups was performed by one-way analysis of variance. The ROC curve of working characteristics of subjects was used to determine the diagnostic cut-off point value of EAT thickness and IMA concentration for coronary heart disease and obtain the corresponding (at the certain diagnostic cut-off point value) sensitivity and specificity. $P<.05$ was considered statistically significant.

\section{RESULTS}

Comparison of clinical baseline data of study patients: There were no significant differences in age, gender, hypertension, diabetes, smoking, and low- or high-density lipoprotein between the groups $(P>.05)$. The total cholesterol and triglyceride levels in the coronary heart disease group (multi-vessel disease group) significantly were higher than those in the normal control group; the difference was statistically significant $(P<.05, P<.01)$. With the coronary heart disease single-vessel disease group and double-vessel disease group, the total cholesterol and triglyceride levels were not significantly different from those in the normal control group $(P>.05)$ (Table 1$)$.

Comparison of EAT thickness and IMA concentration between the coronary heart disease groups and control group: The EAT thickness and IMA concentration in patients with coronary heart disease significantly were higher than those in the control group $(P<.01)$. The EAT thickness and IMA concentration in the single-vessel disease group, doublevessel disease group, and multi-vessel disease group showed an increasing trend, and the difference was statistically significant $(P<.01)$ (Table 2$)$.

Application of EAT thickness and IMA concentration in the diagnosis of coronary heart disease: The area under the ROC curve (AUC value) of the coronary heart disease diagnosis group was 0.785 , among which the AUC values of the single-vessel disease, double-vessel disease, and multi-vessel disease were $0.695,0.812$ and 0.841 , respectively. The thickness of EAT has a certain value in the diagnosis of coronary heart disease, especially in patients with two-vessel disease and multi-vessel disease. ROC curve showed that $6.12 \mathrm{~mm}$ was the best critical point for the diagnosis of coronary heart disease, the corresponding sensitivity was $53.33 \%$, and specificity was $87.6 \%$. The area under ROC curve (AUC value) analysis of IMA concentration showed that the AUC value of the coronary heart disease diagnosis group was 0.845 , and the AUC 
Table 2. Comparison of EAT thickness and IMA concentration between coronary heart disease group and control group

\begin{tabular}{lccc}
\hline Groups & \multicolumn{3}{c}{ AT Thickness } \\
& Cases & $(\mathrm{mm})$ & IMA $(\mathrm{U} / \mathrm{L})$ \\
\hline Coronary heart disease group & 180 & $6.51 \pm 1.22 *$ & $76.12 \pm 14.87^{*}$ \\
Single-vessel disease group & 60 & $5.66 \pm 1.32^{*}$ & $68.12 \pm 13.97 *$ \\
Double-vessel disease group & 60 & $6.24 \pm 1.41 *$ & $76.51 \pm 14.98^{*}$ \\
Multi-vessel disease group & 60 & $6.86 \pm 1.25^{*}$ & $84.86 \pm 15.55 *$ \\
Normal control group & 60 & $4.88 \pm 0.85$ & $54.43 \pm 12.52$ \\
\hline
\end{tabular}

Note: compared with the control group, $* P<.01$.

value of single-vessel disease, double-vessel disease and multivessel disease were $0.713,0.831$, and 0.899 , respectively. The concentration of IMA had a good diagnostic value for the single-vessel disease group, double-vessel disease group, and multi-vessel disease group. The best critical point for ACS diagnosis was 72 U/L of IMA concentration, with the corresponding $76.0 \%$ sensitivity and $72.3 \%$ specificity. Further analysis showed that the sensitivity and specificity of the two methods were $88.3 \%$ and $65.44 \%$, respectively, in parallel test and $40.15 \%$ and $96.89 \%$, respectively, in series test.

\section{DISCUSSION}

The most critical condition in patients with coronary heart disease - acute myocardial infarction can be treated with thrombolysis, intervention and bypass surgery. These measures are conducive to saving patients' lives but compared with the maintenance of coronary blood vessels, early detection of vascular disease is more important. In June 2004, the Ministry of Health approved the early detection of vascular disease detection technology nationwide. The main content calls for the use non-invasive and simple methods to detect subclinical vascular lesions, prompting patients to take effective interventions in time to prevent the occurrence of serious vascular events. In 2005, the International Vascular Health Society was formally established, and vascular health has become a new hot spot of academic attention [Falkner 2010].

The epicardial adipose tissue (EAT) studied in this analysis is deposited on the epicardium and distributed along the coronary artery. It is involved in the secretion of a large number of adiponectin, resistin and other adipokines and inflammatory factors, such as IL- $1 \beta$ and IL-6. The inflammatory response of the coronary intima plays an important role in promoting the development of coronary atherosclerosis and coronary heart disease [Mazzali 2015; Li 2015]. At present, EAT is an independent risk factor for coronary heart disease, and the thickness of EAT is positively correlated with the severity of coronary artery disease. It has a good application value in the diagnosis of coronary heart disease [Yuan 2014; Faghihi 2015]. This study also confirmed that the thickness of EAT in patients with coronary heart disease was significantly higher than that in the normal control group, and the thickness of EAT was positively correlated with the severity of coronary heart disease. The ROC curve analysis of EAT thickness was $6.12 \mathrm{~mm}$, which was the best critical point for the diagnosis of coronary heart disease. The AUC value was 0.785 . The thickness of EAT has certain value in the diagnosis of coronary heart disease.

IMA is a marker of early myocardial ischemia. It begins to rise 5 to 10 minutes after myocardial ischemia and reaches a peak at 3 to 4 hours. It still can last for several hours after ischemic cessation and has a high sensitivity for the diagnosis of coronary heart disease [Yuan 2014]. Recent clinical trials have shown that IMA has important application value in the diagnosis of coronary heart disease, especially in the early stage of myocardial ischemia and myocardial injury, and plays an important role in the diagnosis and risk stratification of coronary heart disease [Song 2008; Gurumurthy 2014; Wang 2015]. The results of this study showed that the IMA concentration in patients with coronary heart disease was significantly higher than that in normal controls, and the IMA concentration positively was correlated with the severity of coronary artery disease in patients with coronary heart disease. The AUC value was 0.845 . Therefore, IMA concentration has certain diagnostic value for coronary heart disease. However, because IMA is susceptible to many factors such as age, genetic variation, race, exercise, and serum albumin levels, its cardiac specificity is poor, and it needs to be combined with other markers for detecting myocardial ischemia and myocardial damage to improve diagnosis accuracy.

To further improve the diagnosis rate of patients with coronary heart disease and to explore the non-invasive detection method for the diagnosis of this disease, this study selected 180 patients with coronary heart disease and 60 healthy medical examinations from May 2017 to December 2018. The combined detection of EAT thickness and IMA concentration showed that the EAT thickness and serum IMA concentration in patients with coronary heart disease were significantly higher than those in the control group, and increased with the rising number of coronary stenosis lesions. The sensitivity, specificity, and misdiagnosis rate of EAT were $53.33 \%$, $87.6 \%, 46.67 \%$ and $12.4 \%$, respectively, and those of IMA were $76.0 \%, 72.3 \%, 24.0 \%$ and $27.7 \%$, respectively. The sensitivity, specificity, missed diagnosis rate and misdiagnosis rate were $88.3 \%, 96.89 \%, 11.7 \%$ and $3.11 \%$, respectively, which greatly improved the sensitivity and accuracy of diagnosis of coronary heart disease.

In summary, ultrasound detection of EAT thickness and IMA concentration has certain reference value for the diagnosis of coronary heart disease. The combined diagnosis of the two significantly can improve the diagnosis rate and accuracy of coronary heart disease. It is of great significance for early detection and treatment of coronary heart disease, prevention of adverse events of the disease, and improvement of prognosis. In addition, there may be differences in EAT thickness and serum IMA concentration in patients with different types of coronary heart disease. It is hoped that more cases will be collected in the future to further explore the clinical application value of the two in the diagnosis of different types of coronary heart disease. 


\section{REFERENCES}

Faghihi S, Vasheghani-Farahani A, Parsaee M, et al. 2015. Association between epicardial fat thickness and premature coronary artery disease: a case control study . Res Cardiovasc Med 23, 4(2): e25679.

Falkner B, Lurbe E, Schaefer F, el a1. 2010. High blood pressure in childrenren: clinical and health policy implications. J Clin Hypertens 12(4): 261-27.

Gurumurthy P, Borra SK, Yeruva RK, et al. 2014. Estimation of Ischemia Modified Albumin (IMA) Levels in Patients with Acute Coronary Syndrome. Indian J Clin Biochem 29(3): 367-371.

Li J, Guo Q, Zhao J, et al. 2015. Correlation between epicardial fat density and coronary plaque. Chinese Journal of Clinical Medical Imaging 26(3): 179-182.

Mazzali G, Fantin F, Zoico E, et al. 2015. Heart fat infiltration in subjects with and without coronary artery disease. Clin Endocrinol Metab 100(9): 3364-3371.

Song X, Lu S. 2008. Evidence-based evaluation of the value of ischemic modified albumin in the diagnosis of myocardial ischemia in patients with coronary heart disease. Chinese Journal of Evidence-based Cardiovascular Medicine 1(1): 49-52.
Wang H. 2010. Vascular medicine: vascular health assessment and comprehensive prevention and treatment of vascular diseases. Chinese Journal of Medicine 90(30): 2092-2093.

Wang P, Zheng X, Wang Y. 2015. Relationship between serum ischemic modified albumin level and coronary artery lesions in patients with acute coronary syndrome. Chinese Journal of Evidence-based Cardiovascular Medicine 7(5): 650- 651.

Wang T, Liu Q, Pu Y, et al. 2014. Predictive value of epicardial adipose tissue for coronary heart disease. Chinese Journal of Arteriosclerosis 22(1): 61-64.

Xia H, Deng Y, Yang H, et al. 2011. Correlation analysis of high frequency ultrasound measurement of epicardial adipose tissue thickness and coronary heart disease risk factors. Chinese Journal of Ultrasonography 20(3): 197-200.

Yan H, Tang Y, Xu J. 2016. Clinical analysis of non-invasive detection of coronary atherosclerosis. Chinese Journal of Evidence-based Cardiovascular Medicine 8(8): 992-994.

Yuan C, Su Y, Ye S. 2014. Echocardiographic determination of epicardial fat thickness in the diagnosis of coronary heart disease. Clinician Medical Engineering, 21(6): 685-686. 